\title{
LIVER
}

\section{Delayed hepcidin response explains the lag period in iron absorption following a stimulus to increase erythropoiesis}

\author{
D M Frazer, H R Inglis, S J Wilkins, K N Millard, T M Steele, G D McLaren, A T McKie, C D Vulpe, \\ G J Anderson
}

See end of article for authors' affiliations .....................

Correspondence to: Dr G J Anderson, Iron Metabolism Laboratory Queensland Institute of Medical Research, PO Royal Brisbane Hospital, Brisbane Queensland 4029, Australia; gregA@ qimr.edu.au

Revised version received 3 March 2004

Accepted for publication 31 March 2004

\begin{abstract}
Introduction: The delay of several days between an erythropoietic stimulus and the subsequent increase in intestinal iron absorption is commonly believed to represent the time required for body signals to programme the immature crypt enterocytes and for these cells to migrate to the villus. Recent data however suggest that signals from the body to alter absorption are mediated by circulating hepcidin and that this peptide exerts its effect on mature villus enterocytes.

Methods: We have examined the delay in the absorptive response following stimulated erythropoiesis using phenylhydrazine induced haemolysis and correlated this with expression of hepcidin in the liver and iron transporters in the duodenum.

Results: There was a delay of four days following haemolysis before a significant increase in iron absorption was observed. Hepatic hepcidin expression did not decrease until day 3, reaching almost undetectable levels by days 4 and 5 . This coincided with the increase in duodenal expression of divalent metal transporter 1, duodenal cytochrome b, and Ireg 1.

Conclusion: These results suggest that the delayed increase in iron absorption following stimulated erythropoiesis is attributable to a lag in the hepcidin response rather than crypt programming, and are consistent with a direct effect of the hepcidin pathway on mature villus enterocytes.
\end{abstract}

A ppropriate body iron stores are maintained by regulated absorption of iron by the duodenum as active regulation of iron excretion appears to be limited. However, the mechanism by which body iron requirements signal changes in the intestinal absorption of iron is unclear. One of the most widely accepted theories is that body signals programme duodenal crypt cells to absorb appropriate levels of iron once they have matured and migrated to the villus. ${ }^{23}$ Central to this hypothesis is the suggestion that there is a 2-3 day lag period between certain stimuli and the subsequent change in iron absorption. This coincides with the time taken for newly formed cells of the crypts to migrate to the villus tip and, therefore, represents the time required for the absorptive cells of the villus to be completely replaced with newly "programmed" cells from crypts.

We have recently re-evaluated the evidence often quoted in support of a role for crypts in the regulation of iron absorption and found that most studies are more appropriately interpreted as indicating a direct signal from the body to mature enterocytes of the villus. ${ }^{4}$ In particular, widely cited papers by Conrad and colleagues ${ }^{5}$ and Charlton and colleagues ${ }^{6}$ show that an intravenous injection of iron will decrease iron absorption maximally within 18-24 hours, far shorter than the 2-3 day lag period required for programming and migration of crypt cells.

Recent molecular evidence also suggests that signals to alter iron absorption have a direct effect on villus enterocytes. Many of these data have resulted from the discovery of hepcidin, a putative humoral regulator of iron homeostasis. Hepcidin is an antimicrobial peptide produced almost exclusively by the liver and secreted into the bloodstream..$^{7-9}$ Hepatic hepcidin expression is increased in iron overload ${ }^{9-11}$ and decreased in iron deficiency ${ }^{9}$ and when erythropoiesis is stimulated. ${ }^{12}$ Importantly, mice lacking hepcidin develop severe iron loading ${ }^{13}$ whereas mice with constitutively high hepcidin expression develop iron deficiency anaemia. ${ }^{14}$ These studies suggest that hepcidin is an inhibitor of iron absorption. We have recently demonstrated that hepcidin expression is decreased in patients with HFE associated haemochromatosis, ${ }^{15}$ suggesting that inappropriate hepcidin expression may underlie the increased iron absorption in this disorder. Mutations in hepcidin have also been implicated in the aetiology of severe iron overload in one form of juvenile haemochromatosis. ${ }^{16}$ Taken together, these studies suggest that hepcidin is the major signal molecule used by the body to regulate iron absorption in response to changes in body iron requirements.

In addition to regulation by body iron needs, hepcidin is increased during an acute phase response (APR). ${ }^{12}$ When an APR is initiated, iron absorption and iron release from the macrophages of the reticuloendothelial (RE) system are decreased, resulting in a reduction in plasma iron concentration. ${ }^{17}$ Recent studies have revealed that hypoferraemia accompanying an APR does not occur in mice lacking hepcidin, indicating that the increase in hepcidin in wildtype mice is responsible for decreased absorption. ${ }^{12}$ This change in iron absorption occurs within hours rather than days ${ }^{17}$ implying that the signal to alter absorption affects mature villus enterocytes. Indeed, we have previously shown that the increase in hepcidin that occurs during an APR is followed within eight hours by a decrease in duodenal expression of iron transport molecules. ${ }^{18}$ In addition, we have shown a close temporal relationship between changes in hepcidin expression and alterations in iron absorption following the switch from an iron replete to an iron deficient diet, ${ }^{19}$ suggesting that hepcidin also exerts its effects on

\footnotetext{
Abbreviations: APR, acute phase response; DAB, 3,3'-diaminobenzidine; Dcytb, duodenal cytochrome b; DMT1, divalent metal transporter 1; GAPDH, glyceraldehyde-3-phosphate dehydrogenase; IRE, iron responsive element; PBS, phosphate buffered saline; PHZ, phenylhydrazine; RE, reticuloendothelial; RPA, ribonuclease protection assay; TfR1, transferrin receptor 1; TfR2, transferrin receptor 2
} 
mature enterocytes during a non-pathogenic response to changes in iron requirements.

The increase in absorption that follows bleeding in both humans and rats however occurs $4-5$ days after blood loss. ${ }^{20}$ This lag period has been interpreted as the time required for the body to programme crypt cells and for these cells to migrate to the villus. Thus one would expect hepcidin expression to decrease several days prior to the change in absorption. We have recently proposed an alternative explanation where the lag period observed after blood loss reflects the time required for the body to detect the need for more iron. ${ }^{4}$ Thereafter, hepcidin production decreases and there is a rapid increase in iron absorption as hepcidin acts on mature villus enterocytes. According to this scenario, any decrease in hepcidin expression would occur several days after the stimulus to increase erythropoiesis and immediately prior to any increase in absorption.

In the current study, we have examined regulation of iron absorption following increased erythropoiesis accompanying phenylhydrazine (PHZ) induced haemolysis. The studies revealed that the delayed increase in iron absorption is due to a lag in the hepcidin response and that once this occurs, changes in duodenal iron transporter expression and iron absorption rapidly follow. These results support our hypothesis that the hepcidin pathway regulates iron absorption by a direct effect on mature villus enterocytes.

\section{METHODS}

\section{Animals and treatment}

Male Sprague-Dawley rats (seven weeks old) were used for all experiments. Rats were allowed unlimited access to a standard pellet diet (Norco Stockfeeds, South Lismore, Australia) and water. To induce haemolysis, rats were administered $100 \mathrm{mg} / \mathrm{kg} \quad \mathrm{PHZ}$ hydrochloride (ICN Biomedicals Australasia, Seven Hills, Australia) $(10 \mathrm{mg} / \mathrm{ml}$ in normal saline, $\mathrm{pH} \sim 7$ ) by intraperitoneal injection. At various time points thereafter, animals were anaesthetised (44 mg/kg ketamine and $8 \mathrm{mg} / \mathrm{kg}$ xylazine) and blood was withdrawn from the abdominal aorta for haematological analysis. Total duodenal enterocytes were isolated as previously described ${ }^{21}$ and snap frozen in liquid nitrogen, as was liver tissue. All experiments described in this study were approved by the Queensland Institute of Medical Research Animal Ethics Committee.

\section{Assessment of iron absorption, haematological parameters, and liver iron status}

Intestinal iron absorption was measured by whole body counting after oral administration of ${ }^{59} \mathrm{Fe}$ in a duplicate series of animals, as previously described. ${ }^{19}$ Haemoglobin and haematocrit were measured with a Cell-Dyn 1600 automated blood analyser (Abbott Laboratories, Brisbane, Australia). Reticulocyte counts were determined by examining blood smears of cells stained with new methylene blue, as described previously. ${ }^{22}$ Liver non-haem iron concentration was measured as previously described. ${ }^{23}$ Enhanced Perls' Prussian Blue stain was performed on formalin fixed paraffin embedded sections using a modification of the method of Wang and colleagues. ${ }^{24}$ Briefly, sections were dewaxed, rehydrated, and incubated in a 1:1 mixture of $4 \%$ potassium ferrocyanide (Ajax Chemicals, Sydney, Australia) and $4 \% \mathrm{HCl}$ for 30 minutes. Sections were then rinsed for five minutes in phosphate buffered saline (PBS) before being incubated for

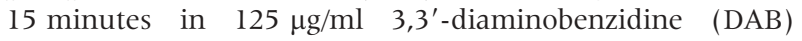
(Sigma-Aldrich, Castle Hill, Australia) in PBS, followed by 15 minutes in fresh DAB solution plus $1.15 \mu \mathrm{l} / \mathrm{ml} 30 \% \mathrm{H}_{2} \mathrm{O}_{2}$. Sections were then rinsed three times in PBS before being counterstained with eosin.

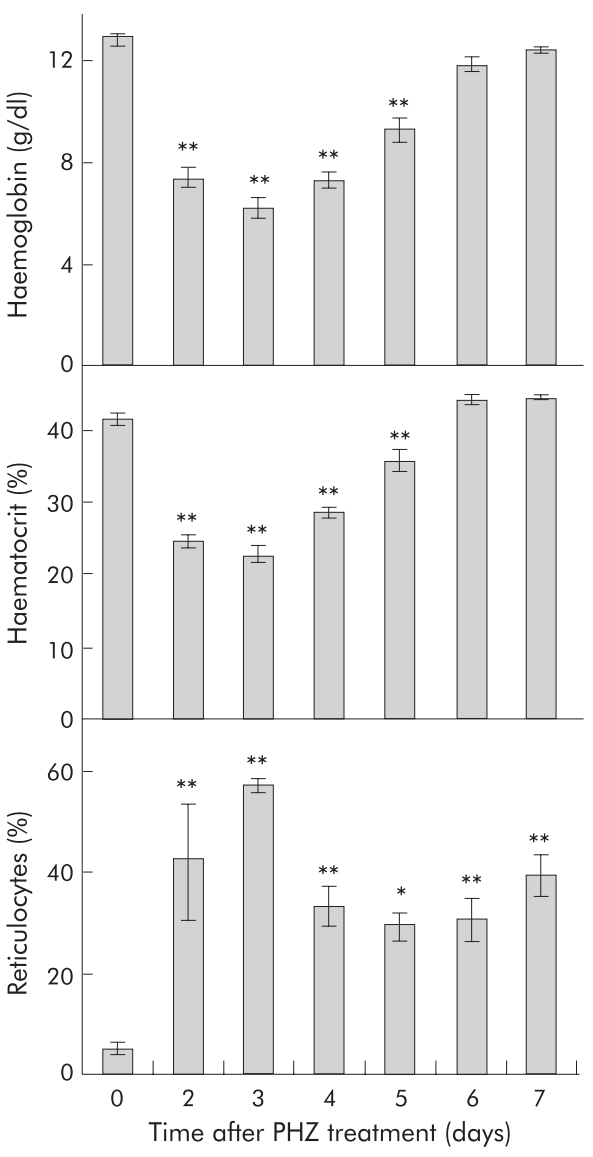

Figure 1 Haematological parameters in rats after phenylhydrazine (PHZ) induced haemolysis. Blood was obtained from rats at various time points following $\mathrm{PHZ}$ induced haemolysis, and haemoglobin

concentration, haematocrit, and reticulocyte count were determined, as described in the methods. Reticulocytes are expressed as a percentage of total blood cells. Data represent the mean (SEM) of five animals.

Statistical significance is shown relative to day $0:{ }^{*} p<0.05,{ }^{* *} p<0.01$.

\section{Analysis of serum transferrin species}

The method used to separate the molecular forms of transferrin in serum was based on previous studies. ${ }^{25-27}$ Briefly, $20 \times$ stock buffer $(2 \mathrm{M}$ Tris, $0.2 \mathrm{M}$ boric acid, $\mathrm{pH}$ 8.4) and urea stock solution $(7.5 \mathrm{M}$, pH 6.0) were deionised using Chelex 100 resin (Bio-Rad Laboratories, Hercules, California, USA) prior to use. Serum was diluted 1 in 9 with rivanol precipitation buffer $(3.75 \mathrm{mg} / \mathrm{ml}$ rivanol, $10 \%$ glycerol in $1 \times$ stock buffer) and mixed by inversion. The solution was incubated for 10 minutes at room temperature before being centrifuged at $16000 \mathrm{~g}$ for one minute. Each sample $(5 \mu \mathrm{l})$ was electrophoresed at $45 \mathrm{~V}$ for 17 hours at $4^{\circ} \mathrm{C}$. The electrophoretic support medium was a $6 \mathrm{M}$ urea and $6 \%$ polyacrylamide gel (37.5:1 acrylamide/bis ratio) prepared in $1 \times$ stock buffer. The electrophoresis running buffer was $1 \times$ stock buffer containing $1.6 \mathrm{mM}$ EDTA. The protein was then transferred to a membrane and subjected to western blotting, as previously described, ${ }^{28}$ using a sheep antihuman transferrin primary antibody (Silenus Laboratories, Hawthorne, Australia) diluted 1 in 1000 and an antisheep horseradish peroxidase conjugated secondary antibody (Silenus Laboratories) diluted 1 in 5000.

RNA extraction and ribonuclease protection assay

Total RNA was extracted from tissue samples using TRIzol reagent (Invitrogen, Melbourne, Australia) as per the manufacturer's instructions. Ribonuclease protection assays 


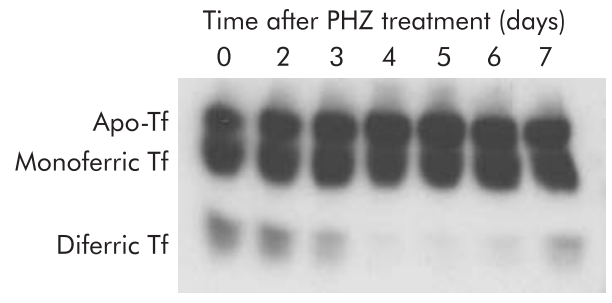

Figure 2 Transferrin (Tf) species in rat serum after phenylhydrazine (PHZ) induced haemolysis. Serum was obtained from rats at various time points following $\mathrm{PHZ}$ induced haemolysis and analysis of the transferrin species present performed by urea gel electrophoresis and western blotting, as described in the methods. A representative gel from five replicates is shown.

(RPAs) were performed as previously described ${ }^{19}{ }^{28}$ using $5 \mu \mathrm{g}$ of total RNA. The housekeeping gene glyceraldehyde-3phosphate dehydrogenase (GAPDH) was used for normalisation.

\section{Western blotting and immunohistochemistry}

Protein was extracted from isolated enterocytes and expression of Iregl and divalent metal transporter l (DMTl) was determined by western blotting, as described previously. ${ }^{19} 28$ Actin was used as a loading control and was detected using a rabbit antiactin antibody (Sigma-Aldrich). Iregl protein was localised by immunofluorescence microscopy using sections cut from duodenal tissue snap frozen in OCT embedding compound (Sakura Finetek USA, Torrance, California, USA), as previously described. ${ }^{19}$ The Iregl antibody was used at a 1 in 100 dilution.

\section{Statistics}

All values are expressed as mean (SEM). Statistical differences between means were calculated using ANOVA followed by Dunnett's post hoc analysis. Statistical analyses were performed using Instat (GraphPad Software, San Diego, California, USA).

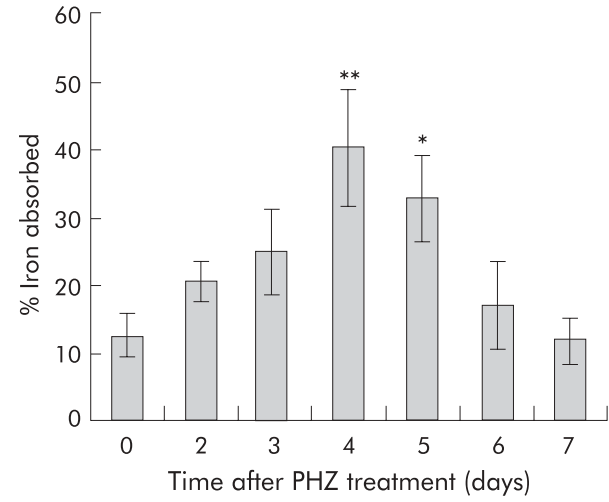

Figure 4 Intestinal iron absorption in rats after phenylhydrazine (PHZ) induced haemolysis. Iron absorption was determined in rats at various time points following PHZ induced haemolysis using radioactive iron, as described in the methods. Absorption is presented as the percentage of the initial iron dose retained by the animals five days after dosing. Data represent the mean (SEM) of 4-10 animals. Statistical significance is shown relative to day $0:{ }^{*} p<0.05,{ }^{* *} p<0.01$.

\section{RESULTS}

\section{Effect of PHZ induced haemolysis on haematological parameters}

Administration of an intraperitoneal dose of PHZ induced rapid haemolysis with haemoglobin levels and haematocrit decreasing to $48 \%$ and $55 \%$ of control levels, respectively, after three days (fig 1). These parameters showed a progressive recovery after day 3 with both haemoglobin levels and haematocrit reaching control values by day 6 .

The reticulocyte percentage increased dramatically within two days after PHZ treatment, consistent with the expected increase in erythropoiesis, although there was substantial inter-animal variation at this time point (range 20-79\%). By day 3, the reticulocyte count had peaked at a mean level 11.6-fold higher than controls. At this time point, the reticulocyte count was consistently elevated in all rats (range 52-60\%). Reticulocyte levels declined after day 3 but

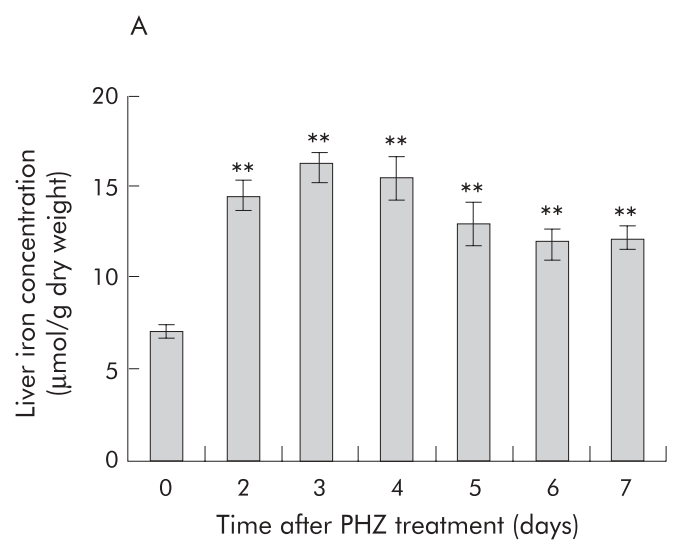

B

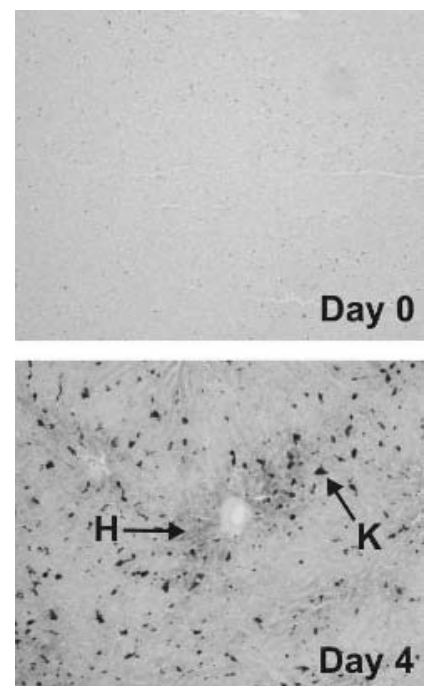

Figure 3 Liver iron status in rats after phenylhydrazine (PHZ) induced haemolysis. Liver tissue was obtained from rats at various time points following $\mathrm{PHZ}$ induced haemolysis and hepatic iron concentration (A) was determined, as described in the methods. Liver tissue from day 0 and day 4 animals was fixed in formalin, sectioned, and subject to enhanced Perls' stain (B), as described in the methods. A representative section demonstrating Kupffer cell $(K)$ and hepatocyte $(H)$ stainable iron is shown $(n=5)$. Data represent the mean $(S E M)$ of five animals. Statistical significance is shown relative to day $0:{ }^{* *} p<0.01$. 

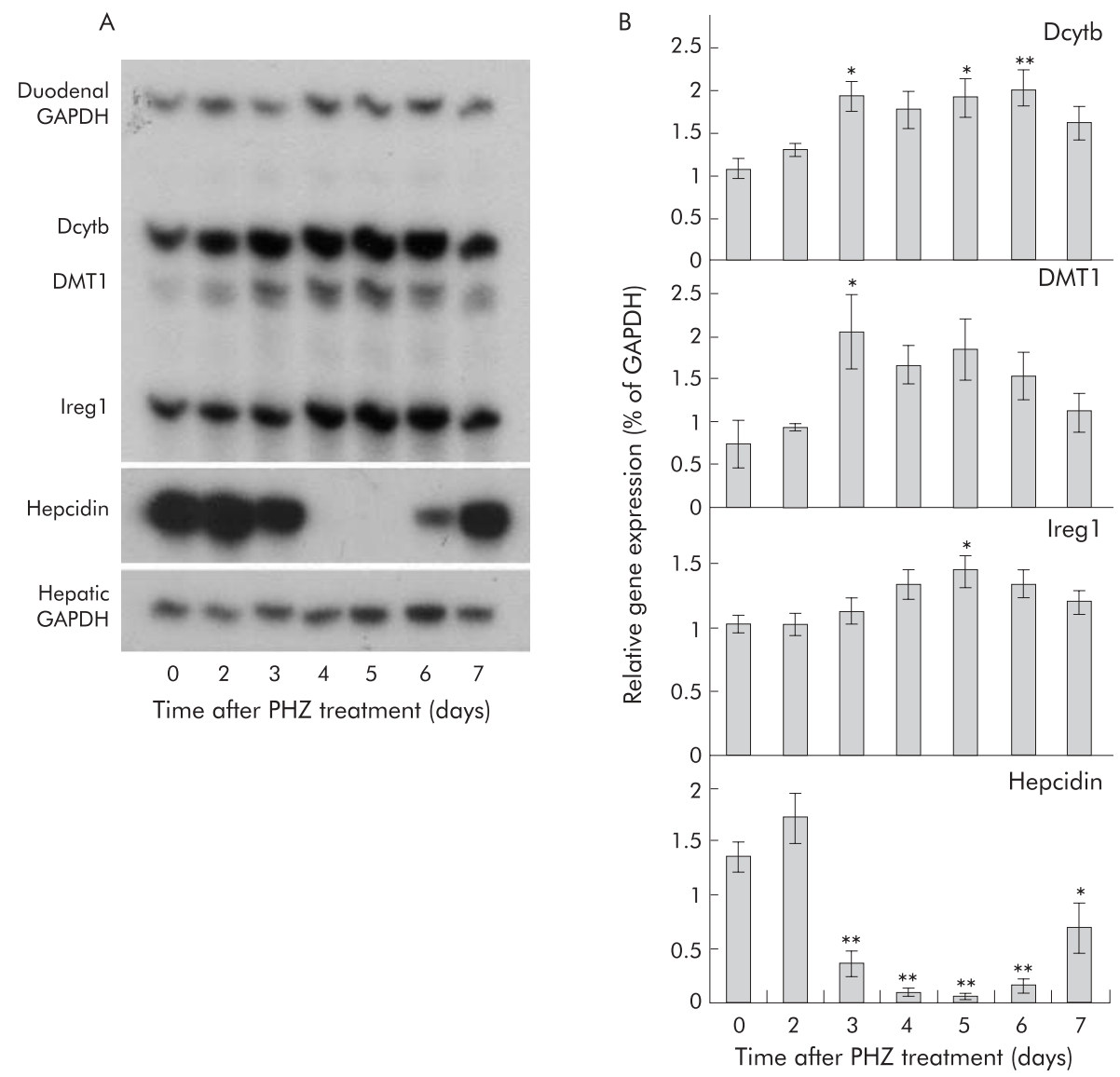

Figure 5 Gene expression in rats after phenylhydrazine (PHZ) induced haemolysis. Duodenal enterocytes and liver tissue were isolated from rats at various time points following PHZ induced haemolysis. Total RNA was extracted and gene expression determined by ribonuclease protection assay (RPA) using $5 \mu \mathrm{g}$ of RNA, as described in the methods. Representative RPAs are shown for each gene (A). Band intensities were quantitated by densitometry, corrected for loading using GAPDH as a control, and graphed as a proportion of GAPDH (B). Data represent the mean (SEM) of five animals. Statistical significance is shown relative to day $0:{ }^{*} \mathrm{p}<0.05,{ }^{* *} \mathrm{p}<0.01$. Dcytb, duodenal cytochrome b; DMT1, divalent metal transporter 1; GAPDH, glyceraldehyde-3-phosphate dehydrogenase.

remained significantly higher than control levels seven days after treatment.

Examination of transferrin species in the serum of $\mathrm{PHZ}$ treated rats by western blotting revealed a distinct decrease in diferric transferrin three days after haemolysis, reaching almost undetectable levels on days 4 and 5 (fig 2). Diferric transferrin then began to recover on days 6 and 7. No major changes in apotransferrin and monoferric transferrin were observed over the course of the experiment.

\section{Hepatic iron stores and intestinal iron absorption}

Hepatic non-haem iron concentrations increased markedly after PHZ treatment, reaching a maximum 2.3 times that of control animals within three days (fig 3A). Thereafter, liver iron levels appeared to decrease somewhat but remained significantly higher than control levels seven days after haemolysis. Enhanced Perls' stain of formalin fixed liver sections from day 4 revealed prominent iron deposits, primarily in Kupffer cells (fig 3B). Iron levels were also increased in hepatocytes surrounding portal tracts. No iron deposits were detected in control animals.

To examine the effect of increased erythropoietic activity on intestinal iron absorption, absorption was measured in PHZ treated and control rats. Absorption was significantly increased (3.2-fold compared with controls) four days following injection of PHZ, as shown in fig 4. Absorption remained significantly increased until day 5 , then declined to control levels.

\section{Expression of genes of iron metabolism following PHZ} induced haemolysis

The results described above indicate that our model of stimulated erythropoiesis successfully induced an increase in intestinal iron absorption. Moreover, our results are consistent with the previously reported lag period of approximately four days ${ }^{20}$ between the haemolytic stimulus and change in absorption. We next examined whether changes in expression of hepcidin coincided with the change in absorption or whether they preceded the absorption change by several days, as predicted by the crypt programming model. We also examined duodenal expression of genes known or proposed to be involved in iron absorption.

RPA analysis (fig 5) showed that hepcidin mRNA expression was unchanged on the first two days following haemolysis. Thereafter, however, expression decreased dramatically, reaching almost undetectable levels by days 4 and 5. This was followed by a recovery in expression reaching $52 \%$ of control levels by day 7. This expression pattern matched closely the observed change in absorption described above. The reduction in hepcidin expression was accompanied by a similarly transient increase in expression of mRNAs encoding the brush border iron transporter DMTl, ${ }^{29}{ }^{30}$ the brush border ferrireductase duodenal cytochrome b (Dcytb), ${ }^{31}$ and the putative basolateral iron transporter Iregl..$^{32}{ }^{33}$ Only the iron responsive element (IRE) containing splice variant of DMTl is shown, as the non-IRE form is expressed at much lower levels in the gut. ${ }^{28}$ Similar increases in DMTl protein were 

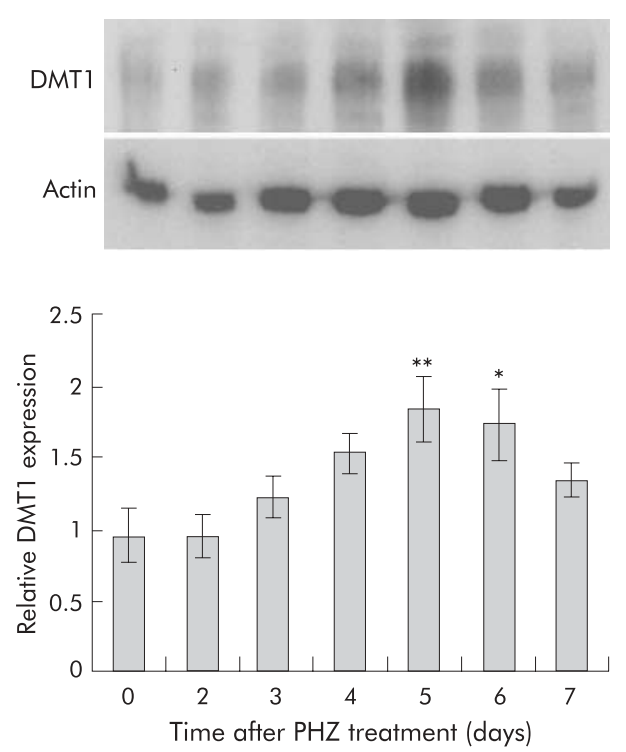

Figure 6 Divalent metal transporter 1 (DMT1) protein expression in rats after phenylhydrazine (PHZ) induced haemolysis. Duodenal enterocytes were isolated from rats at various time points, as described in the methods. Protein was extracted from enterocytes and analysed by western blotting using antibodies specific to DMT1 and actin. A representative blot is shown above. Band intensities were quantitated by densitometry, corrected for loading using actin as a control and graphed as a proportion of actin. Data represent the mean (SEM) of five animals. Statistical significance is shown relative to day 0 : ${ }^{*} p<0.05,{ }^{* *} p<0.01$.

also observed (fig 6). Iregl protein expression followed the same trend as the other molecules with levels peaking 4-5 days after PHZ induced haemolysis although the difference did not reach statistical significance (fig 7A). Further examination of Ireg 1 protein by immunofluorescence however demonstrated markedly increased expression of the protein on the basolateral surface of duodenal enterocytes four days after PHZ treatment compared with control animals (fig 7B). Negative controls (without primary antibody) showed no staining (data not shown).

\section{DISCUSSION}

The current work confirms and extends the results of previous studies that have demonstrated a lag period of approximately four days after an erythropoietic stimulus before intestinal iron absorption increases. ${ }^{20}$ This delay is proposed to represent the time taken for immature enterocytes of the crypts to receive signals from the body and then migrate to the villus and differentiate into absorptive cells. However, recent molecular studies have provided evidence that signals to alter iron absorption are mediated by hepcidin and have a direct effect on mature enterocytes (for review, see Frazer and Anderson ${ }^{4}$ ). The delay in absorption following stimulated erythropoiesis is still consistent with such a direct action if the delay represents the time required by the body to detect the need for more iron and alter hepcidin expression. The studies described in this paper were conducted to investigate this possibility by using PHZ induced haemolysis to stimulate erythropoiesis in a rat model.

We observed no change in expression of hepcidin until three days after administration of PHZ, with the lowest levels evident 4-5 days after haemolysis was induced. This coincided with the highest levels of absorption and with peak expression of molecules involved in intestinal iron transport. The close temporal relationship between changes in hepcidin expression and changes in intestinal iron absorption is consistent with the concept that the lag period following a stimulus to increase erythropoiesis represents the time required for the body to assess iron needs. ${ }^{4}$ Once the requirement for more iron is recognised, hepcidin expression is decreased, resulting in a rapid increase in iron transporter expression and hence iron absorption. This sequence of
A

Ireg

Actin
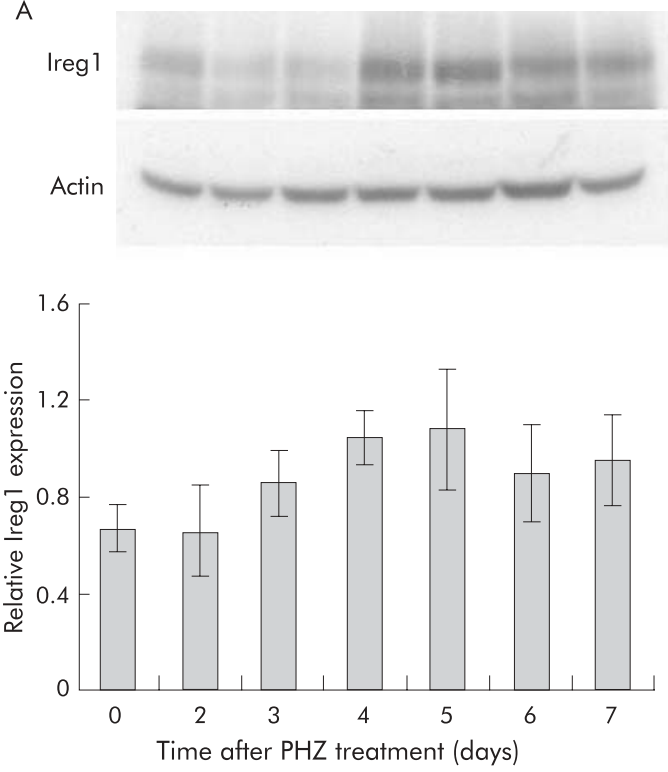
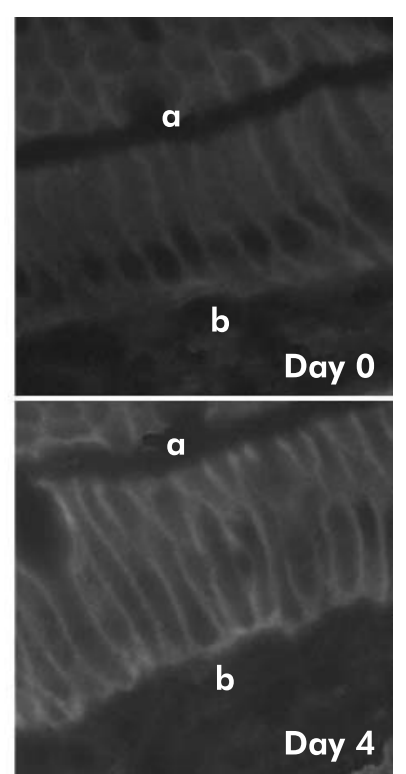

Figure 7 Iregl protein expression in rats after phenylhydrazine (PHZ) induced haemolysis. Duodenal enterocytes were isolated from rats at various time points, as described in the methods. Protein was extracted from enterocytes and analysed by western blotting using antibodies specific to Ireg 1 and actin. A representative blot is shown (A). Band intensities were quantitated by densitometry, corrected for loading using actin as a control and graphed as a proportion of actin. Duodenal sections from day 0 and day 4 animals were analysed by immunofluorescence using antibodies specific for Iregl, as described in the methods. Representative sections are shown indicating the apical (a) and basal (b) side of the epithelium (B). Data represent the mean (SEM) of five animals. Despite the increase in Iregl, no statistically significant difference was observed between any treatment group and day 0 animals. 
events is not compatible with the crypt programming hypothesis.

The results of the current study agree with our previous data showing that changes in hepcidin expression are closely associated with changes in iron transporter expression and iron absorption. This was the case following both the change from a control to an iron deficient $\operatorname{diet}^{19}$ and after induction of an APR. ${ }^{18}$ In the latter investigation, we found that DMT1 and Dcytb expression decreased within eight hours of an increase in hepcidin. In the current study, this close relationship between hepcidin expression and absorption was observed both in response to PHZ and during subsequent recovery. These data provide further evidence that hepcidin acts to increase or decrease absorption via an effect on villus enterocytes. It is interesting to note that, in the current study, we observed only small increases in levels of Iregl mRNA and protein but a significant increase in the amount of Iregl on the basolateral membrane, supporting previous suggestions that post-translational events are important in the regulation of Iregl mediated iron export. ${ }^{34}$ In addition to changes in transporter expression, it has previously been demonstrated that changes in membrane potential and villus length in the duodenum accompany PHZ induced haemolysis and such changes could also contribute to the observed increase in iron absorption. ${ }^{35}$

It has been shown that expression of hepcidin is influenced by both body iron stores and the rate of erythropoiesis ${ }^{9-12}$ but it is not known how the body detects the need for more iron and regulates hepcidin expression accordingly. The existence of a "stores regulator" function has been proposed as a mechanism that is responsive to the body's iron stores, increasing absorption when stores are low and decreasing absorption when the body is iron replete. ${ }^{36}$ Similarly, a proposed "erythroid regulator" appears to adjust iron absorption in response to the iron requirements of the bone marrow, functioning to ensure that an adequate supply of iron is maintained for erythropoiesis by increasing iron absorption when erythropoiesis is stimulated and the demand for iron is high. ${ }^{36}$ On the basis of current evidence, it is likely that the effects of both the stores and erythroid regulators on iron absorption are mediated by hepcidin. We have recently suggested that these regulators may signal changes in hepcidin by altering the ratio of diferric transferrin to transferrin receptor 1 (TfRl) levels in the body. ${ }^{4}$ In this model, increased TfRl levels in the marrow resulting from stimulation of erythropoiesis would create an increased demand for iron resulting in decreased diferric transferrin levels, which would then be detected by the HFE/ TfR 1 complex and TfR 2 in the liver. The signals produced by these molecules would trigger a decrease in hepcidin expression and a subsequent increase in iron absorption. In contrast, an increase in iron stores would shift the ratio in the other direction by reducing TfRl expression and increasing diferric transferrin levels, thereby increasing hepcidin expression and decreasing absorption.

The results of the current study are consistent with this model. We show that the decrease in hepcidin expression occurs despite increased macrophage and hepatocyte iron loading, a condition that normally leads to an elevation in hepcidin. ${ }^{9-11}$ The increased cellular iron content would be expected to decrease hepatic TfRl expression. However, red blood cell precursors in the bone marrow express high levels of TfRl in order to supply the iron required for haemoglobin production. ${ }^{37}$ Studies have demonstrated that TfRl preferentially binds diferric transferrin ${ }^{38}$ and that at normal transferrin saturation, most of the iron taken up will be in the form of diferric transferrin. ${ }^{39}$ Thus the increase in reticulocytes observed on days 2 and 3 would be expected to be accompanied by a dramatic decrease in the level of diferric transferrin in the circulation. This was indeed the case. Changes in diferric transferrin observed in the current study correlated closely with changes in hepcidin expression. The resulting increase in iron absorption and RE cell iron output over the next few days, coupled with a slowing in the rate of erythropoiesis and a diminishing demand for iron associated with a return of the blood haemoglobin concentration to normal, would lead to an increase in diferric transferrin levels, as we observed. According to our model, this would trigger the recovery of hepcidin expression towards baseline levels that was observed on days 6 and 7. It is unlikely that changes in diferric transferrin levels per se alter iron absorption, as in patients with hepcidin mutations both transferrin saturation and intestinal absorption are greatly elevated. ${ }^{40}$

An intriguing observation in the current study is the return of iron absorption to normal by day 7 following PHZ treatment, despite incomplete recovery of hepcidin expression. The fact that reticulocyte counts remained high at this time suggests that erythropoiesis, and hence iron demand, was still elevated in these animals, albeit not to the same extent seen at 3-4 days after PHZ treatment. A likely explanation for this phenomenon is the continued presence of an increased number of erythroid precursors still maturing in the marrow, following the initial expansion of the erythroid mass in response to the episode of haemolysis one week earlier. This is consistent with intermediate hepcidin levels seen on day 7. Persistence of reticulocytosis despite a return of absorption to normal implies that RE system iron output remains increased at a level that is adequate to satisfy the increased erythropoietic demand. Thus it appears that small to modest reductions in hepcidin levels are sufficient to stimulate iron release from the RE system but that stimulation of intestinal iron absorption requires a further decrease until levels fall below a certain threshold. Such differential regulation would allow the body to modulate iron output from $\mathrm{RE}$ cells in response to alterations in body iron demand (particularly iron supply to the erythroid marrow) without invoking changes in absorption so that more iron can be made available without increasing total body iron load. This may explain the fact that haemolytic anaemias in which erythropoiesis remains effective (for example, chronic haemolytic states such as hereditary spherocytosis) are not typically accompanied by increased iron absorption and do not usually lead to iron overload. ${ }^{41}$ However, in haemolytic conditions associated with increased ineffective erythropoiesis, such as $\beta$-thalassaemia, the increased RE system output cannot adequately supply iron to the greatly expanded erythroid mass and absorption increases as a result. ${ }^{41-43}$ One would predict that hepcidin expression would be lower in the latter case. This is supported by recent evidence demonstrating that thalassaemic mice express lower than expected levels of hepcidin. ${ }^{44}$

In conclusion, the current study supports the proposal that the hepcidin pathway exerts a direct effect on the mature villus enterocytes of the duodenum and suggests that the time lag between a stimulus to increase erythropoiesis and the subsequent increase in iron absorption represents the time required by the body to detect the need for more iron. In the case of haemolytic anaemia, this represents the time between the onset of anaemia and the ensuing increase in erythropoiesis. Once the increased need for iron has been recognised, there is a decrease in hepatic hepcidin expression, followed rapidly by an increase in iron absorption. The decrease in hepcidin expression observed in this study occurred shortly after the onset of increased iron demand by the marrow, supporting our proposal that the amount of plasma diferric transferrin plays a central role in the regulation of body iron homeostasis. 


\section{ACKNOWLEDGEMENTS}

This work was supported in part by grants from the National Health and Medical Research Council of Australia, the National Institute of Diabetes and Digestive and Kidney Diseases (R01 DK-57800), and the Human Frontier Science Program (RGY0328/2001-M).

\section{Authors' affiliations}

D M Frazer*, H R Inglis*, S J Wilkins, K N Millard, T M Steele,

G J Anderson, Iron Metabolism Laboratory, the Queensland Institute of Medical Research and the University of Queensland, PO Royal Brisbane Hospital, Brisbane, Queensland, Australia G D McLaren, Division of Haematology/Oncology, University of California, Irvine, Irvine, California, USA, and VA Long Beach Healthcare System, Long Beach, California, USA

A T McKie, Division of Life Sciences, King's College, London, UK C D Vulpe, Department of Nutritional Sciences and Toxicology, University of California, Berkeley, Berkeley, California, USA

*D M Frazer and H R Inglis contributed equally to the manuscript.

\section{REFERENCES}

1 McCance RA, Widdowson EM. Absorption and excretion of iron. Lancet 1937;ii: 680-4.

2 Lombard M, Chua E, O'Toole P. Regulation of intestinal non-haem iron absorption. Gut 1997;40:435-9.

3 Andrews NC. Disorders of iron metabolism. N Engl J Med 1999:341:1986-95.

4 Frazer DM, Anderson GJ. The orchestration of body iron intake: how and where do enterocytes receive their cues? Blood Cells Mol Dis 2003;30:288-97.

5 Conrad ME, Weintraub LR, Crosby WH. The role of the intestine in iron kinetics. J Clin Invest 1964;43:963-74.

6 Charlton RW, Jacobs P, Bothwell TH. The role of the intestinal mucosa in iron absorption. J' Clin Invest 1965;44:543-54.

7 Krause A, Neitz S, Mägert H, et al. LEAP-1, a novel highly disulfide-bonded human peptide, exhibits antimicrobial activity. FEBS Lett 2000;480:147-50.

8 Park $\mathrm{CH}$, Valore EV, Waring AJ, et al. Hepcidin, a urinary antimicrobial peptide synthesized in the liver. J Biol Chem 2001;276:7806-10.

9 Pigeon C, llyin G, Courselaud B, et al. A new mouse liver-specific gene, encoding a protein homologous to human antimicrobial peptide hepcidin, is overexpressed during iron overload. J Biol Chem 2001:276:7811-19.

10 Ahmad KA, Ahmann JR, Migas, MC, et al. Decreased liver hepcidin expression in the Hfe knockout mouse. Blood Cells Mol Dis 2002;29:361-6.

11 Mazur A, Feillet-Coudray C, Romier B, et al. Dietary iron regulates hepatic hepcidin 1 and 2 mRNAs in mice. Metabolism 2003;52:1229-31.

12 Nicolas G, Chauvet C, Viatte L, et al. The gene encoding the iron regulatory peptide hepcidin is regulated by anemia, hypoxia, and inflammation. J Clin Invest 2002;110:1037-44.

13 Nicolas G, Bennoun M, Devaux I, et al. Lack of hepcidin gene expression and severe tissue iron overload in upstream stimulatory factor 2 (USF2) knockout mice. Proc Natl Acad Sci U S A 2001;98:8780-5.

14 Nicolas $G$, Bennoun $M$, Porteu $A$, et al. Severe iron deficiency anemia in transgenic mice expressing liver hepcidin. Proc Natl Acad Sci U S A 2002; $99: 4596-601$

15 Bridle KR, Frazer DM, Wilkins SJ, et al. Failure of hepcidin upregulation in HFE-associated haemochromatosis implicates the liver in the regulation of body iron homeostasis. Lancet 2003;361:669-73.

16 Roetto A, Papanikolaou G, Politou M, et al. Mutant antimicrobial peptide hepcidin is associated with severe juvenile hemochromatosis. Nat Genet 2003;33:21-2.

17 Cortell S, Conrad ME. Effect of endotoxin on iron absorption. Am J Physiol 1967;213:43-7.
18 Anderson GJ, Frazer DM, Wilkins SJ, et al. Relationship between intestinal iron-transporter expression, hepatic hepcidin levels and the control of iron absorption. Biochem Soc Trans 2002;30:724-6

19 Frazer DM, Wilkins SJ, Becker EM, et al. Hepcidin expression inversely correlates with the expression of duodenal iron transporters and iron absorption in rats. Gastroenterology 2002;123:835-44.

20 Weintraub LR, Conrad ME, Crosby WH. The significance of iron turnover in the control of iron absorption. Blood 1964;24:19-24.

21 Anderson GJ, Powell LW, Halliday JW. The endocytosis of transferrin by rat intestinal epithelial cells. Gastroenterology 1994;106:414-22.

22 Dacie JV, Lewis SM. Practical haematology. Edinburgh: Churchill Livingstone, 1975.

23 Torrance JD, Bothwell TH. A simple technique for measuring storage iron concentrations in formalinised liver samples. S Afr J Med Sci 1968;33:9-11.

24 Wang XS, Ong WY, Connor JR. Increase in ferric and ferrous iron in the rat hippocampus with time after kainate-induced excitotoxic injury. Exp Brain Res 2002;143:137-48.

25 Makey DG, Seal US. The detection of four molecular forms of human transferrin during the iron binding process. Biochim Biophys Acta 1976;453:250-6.

26 Evans RW, Williams J. The electrophoresis of transferrins in urea/ polyacrylamide gels. Biochem J 1980;189:541-6.

27 Davy P, Bingham D, Walters G, et al. Estimation of the saturation of serum transferrin by an electrophoretic technique. Ann Clin Biochem 1982;19:57-9.

28 Frazer DM, Vulpe CD, McKie AT, et al. Cloning and gastrointestinal expression of rat hephaestin: relationship to other iron transport proteins. Am J Physiol 2001;281:G931-9.

29 Gunshin H, Mackenzie B, Berger UV, et al. Cloning and characterization of a mammalian proton-coupled metal-ion transporter. Nature 1997:388:482-8.

30 Fleming MD, Trenor III CC, Su MA, et al. Microcytic anaemia mice have a mutation in Nramp2, a candidate iron transporter gene. Nat Genet 1997:16:383-6.

31 McKie AT, Barrow D, Latunde-Dada GO, et al. An iron-regulated ferric reductase associated with the absorption of dietary iron. Science 2001;291:1755-9.

32 McKie AT, Marciani P, Rolfs A, et al. A novel duodenal iron-regulated transporter, IREG1, implicated in the basolateral transfer of iron to the circulation. Mol Cell 2000;5:299-309.

33 Donovan A, Brownlie A, Zhou Y, et al. Postional cloning of zebrafish ferroportin 1 identifies a conserved vertebrate iron exporter. Nature 2000;403:776-81.

34 Abboud S, Haile DJ. A novel mammalian iron-regulated protein involved in intracellular iron metabolism. J Biol Chem 2000;275:19906-12.

35 O'Riordan DK, Sharp P, Sykes RM, et al. Cellular mechanisms underlying the increased duodenal iron absorption in rats in response to phenylhydrazineinduced haemolytic anaemia. 1995;25:722-7.

36 Finch C. Regulators of iron balance in humans. Blood 1994;84:1697-702.

37 Enns CA, Shindelman JE, Tonik SE, et al. Radioimmunochemical measurement of the transferrin receptor in human trophoblast and reticulocyte membranes with a specific anti-receptor antibody. Proc Natl Acad Sci U S A $1981 ; 78: 4222-5$

38 Young SP, Bomford A, Williams R. The effect of the iron saturation of transferrin on its binding and uptake by rabbit reticulocytes. Biochem $J$ 1984;219:505-10.

39 Huebers H, Csiba E, Huebers E, et al. Molecular advantage of diferric transferrin in delivering iron to reticulocytes: a comparative study. Proc Soc Exp Biol Med 1985;179:222-6.

40 Camaschella C, Roetto A, De Gobbi M. Juvenile hemochromatosis. Semin Hematol 2002;39:242-8.

41 Erlandson ME, Waldon B, Stern G, et al. Studies on congenital hemolytic syndromes. IV. Gastrointestinal absorption of iron. Blood 1962:19:359-78.

42 Pootrakul P, Wattanasaree J, Anuwatanakulchai MS, et al. Increased red blood cell protoporphyrin in thalassemia: a result of relative iron deficiency. Am J Clin Pathol 1984;82:289-93.

43 Cazzola M, Pootrakul P, Bergamaschi G, et al. Adequacy of iron supply for erythropoiesis: in vivo observations in humans. J Lab Clin Med 1987:110:734-9.

44 Adamsky K, Weizer O, Amariglio N, et al. Decreased hepcidin mRNA expression in thalassemic mice. B J Haematol 2004; 124:123-5. 